\title{
Medical leaders urge system change to reduce physician burnout
}

\author{
Cite as: CMAJ 2018 November 5;190:E1312-3. doi: 10.1503/cmaj.109-5684
}

Posted on cmajnews.com on Oct. 15, 2018.

mproving the health and wellbeing of physicians is "now more pressing than ever," said Canadian Medical Association (CMA) President Dr. Gigi Osler at the International Conference on Physician Health in Toronto.

One in four Canadian physicians reports high levels of burnout and one in three screens positive for depression. South of the border, more than half of physicians in the United States have symptoms of burnout. One in five is thinking about reducing their hours and 1 in 50 is considering early retirement, despite mounting physician shortages. Meanwhile, some 400 physicians and 100 medical students die by suicide each year.

"We have to fix this," said American Medical Association President Dr. Barbara McAneny. But it's not enough to teach doctors coping skills, she said. "I don't want yoga at lunch; I want a system that's worth my efforts."

Increasing bureaucracy is undermining physicians' freedom and satisfaction in their work, McAneny explained. "For every hour a physician gets to spend talking to a patient, they spend two hours tapping on a computer."

Compliance and quality improvement efforts have added to that paperwork, noted British Medical Association President Dr. Dinesh Bhugra. Meanwhile, "the practice of medicine has become incredibly complex and much more mechanical."

According to Dr. Anthony Suchman, a health services researcher at Rochester University, the current system is "perfectly designed to cause burnout."

Even the way many people think about health organizations as machines "creates these expectations of external con- trol that are unrealistic and create a lot of anxiety," he said. It may be more helpful to approach changing health systems like changing a conversation, "by participating in it differently."

That means paying attention to patterns in how people relate to each other within an organization and taking small, consistent steps to shift that narrative, Suchman explained. "Anything you think of as a big pattern are just an amalgam of small things happening moment to moment, so if we want to change the culture of an organization we have to start right here, right now."
Change can start with a single person speaking out about a problem and finding a few other people who feel the same to share experiments and solutions, he said.

At Western University, internal medicine residents used the results of a wellness survey as a spark for more meaningful conversations among trainees. "The easiest events to create are fun and relaxing, but if that's all we do the benefits tend to be transient and superficial," said Dr. Linda Wang, who helped to organize a wellness week at Western, including a half-day debriefing event focused on sharing personal stories and experiences. "It is

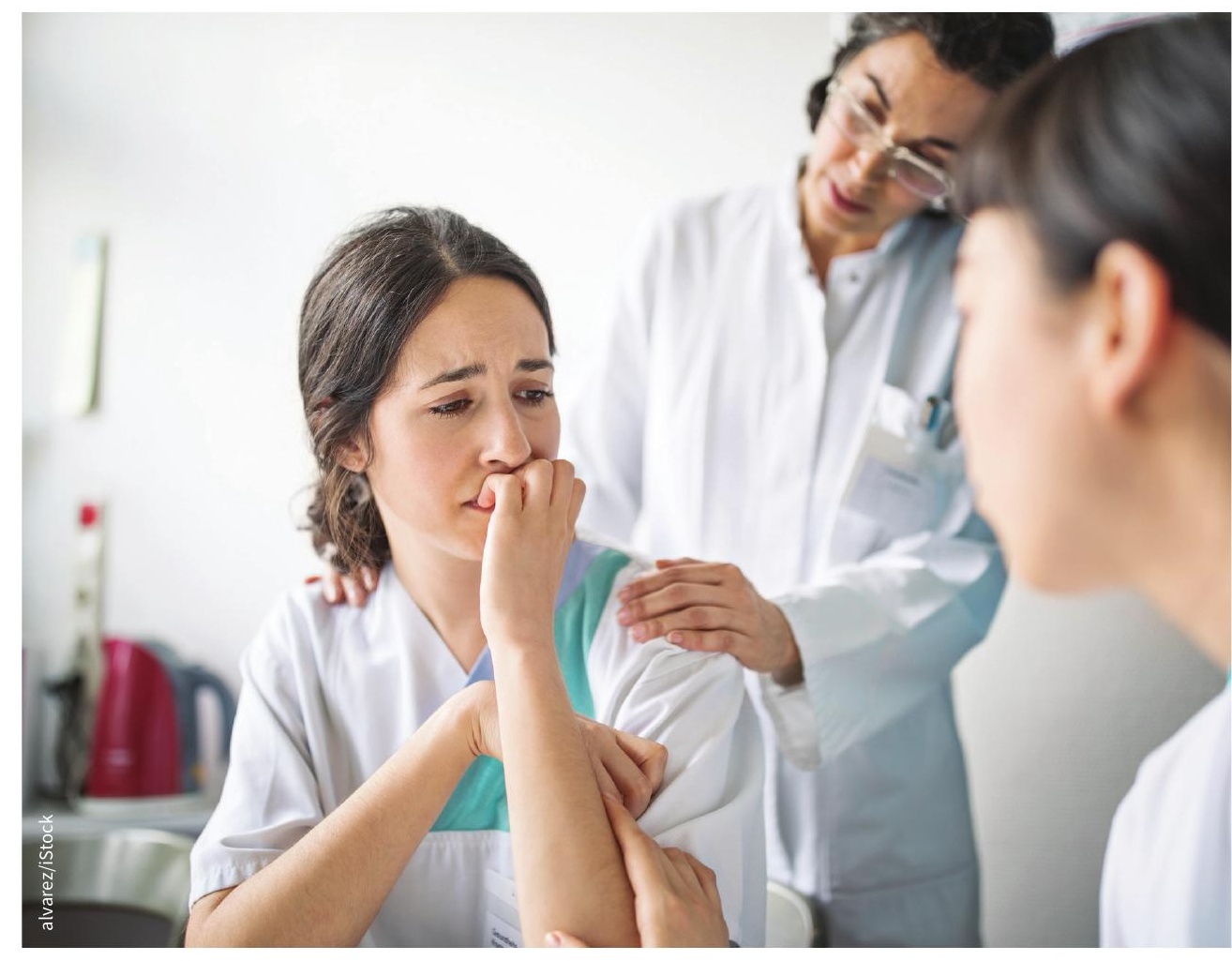

Physician burnout topped the agenda at the International Conference on Physician Health. 
being vulnerable with each other that builds trust, real relationships and community so we're not all just alone working together."

At the debriefing event, Wang shared the results of a survey of Western's internal medicine residents that found $84 \%$ had felt inadequate compared to peers and about half had felt bullied or intimidated on the job. Two consultants also shared stories about similar struggles in their own careers.

All the participants "agreed or strongly agreed they gained perspective, felt more comfortable talking to colleagues about difficult experiences and would like this initiative to continue," Wang said. "Ultimately, we hope to foster an environment where these formal opportunities are not necessary."

In a recent statement, the CMA called on physicians, organizations and systems to share responsibility for promoting a "vibrant and engaged profession."

For individual doctors, this means committing to self-care and supporting peers. The medical profession must also commit to fostering a culture of wellness, which would include eliminating stigma, policies and practices that prevent doctors from seeking help. Meanwhile, health systems and organizations have a respon- sibility to develop and promote supports, including occupational health standards for physicians like those afforded other workers.

For its part, the CMA has created a new department of physician health, with a dedicated vice president and increased resources. The association also recently launched a wellness ambassador program for trainees and is working with the Royal College of Physicians and Surgeons of Canada to strengthen accreditation standards for wellness in medical schools and residency programs.

Lauren Vogel, CMAJ 